Meta

Journal des traducteurs

Translators' Journal

\title{
Modèles explicatifs de la créativité en traduction
}

\section{Ioana Balacescu et Bernd Stefanink}

Volume 48, numéro 4, décembre 2003

URI : https://id.erudit.org/iderudit/008723ar

DOI : https://doi.org/10.7202/008723ar

Aller au sommaire du numéro

\section{Éditeur(s)}

Les Presses de l'Université de Montréal

ISSN

0026-0452 (imprimé)

1492-1421 (numérique)

Découvrir la revue

Citer cet article

Balacescu, I. \& Stefanink, B. (2003). Modèles explicatifs de la créativité en traduction. Meta, 48(4), 509-525. https://doi.org/10.7202/008723ar

\section{Résumé de l'article}

La créativité fait partie des facultés que le traducteur doit mettre en action quotidiennement. Pourtant elle a été consciemment écartée de la recherche en traductologie comme " non saisissable rationnellement "

(Gerzymisch-Arbogast/Mudersbach 1998 : 16), ou utilisée pour légitimer des abus traductologiques inconciliables avec la déontologie du traducteur (" manipulation school »). L'état des recherches actuelles fait apparaître deux facteurs qui, respectivement, justifient et rendent possible une étude de la créativité en traduction. Ce sont, d'une part, l'abandon de la poursuite du fantôme d'une " objectivité " mal comprise au profit d'une exigence de " plausibilité interindividuelle » en traductologie, et, d'autre part, l'apport conjoint de différentes sciences parallèles, comme la psychologie et la linguistique cognitive, qui concourent toutes à mieux servir cette « plausibilité interindividuelle » et qui, pour la première fois, font accéder la traductologie au rang de science interdisciplinaire, une interdisciplinarité qui, par beaucoup de chercheurs, est considérée comme un critère sine qua non pour accéder au statut de science.
Ce document est protégé par la loi sur le droit d'auteur. L’utilisation des services d’Érudit (y compris la reproduction) est assujettie à sa politique d'utilisation que vous pouvez consulter en ligne.

https://apropos.erudit.org/fr/usagers/politique-dutilisation/ 


\title{
Modèles explicatifs de la créativité en traduction
}

\author{
IOANA BALACESCU ${ }^{1}$ \\ Université de Craiova, Craiova, Roumanie \\ ioanadi@hotmail.com \\ BERND STEFANINK \\ Université de Bielefeld, Bielefeld, Allemagne \\ bstefanink@hotmail.com
}

\begin{abstract}
RÉSUMÉ
La créativité fait partie des facultés que le traducteur doit mettre en action quotidiennement $^{2}$. Pourtant elle a été consciemment écartée de la recherche en traductologie comme «non saisissable rationnellement» (Gerzymisch-Arbogast/Mudersbach 1998: 16), ou utilisée pour légitimer des abus traductologiques inconciliables avec la déontologie du traducteur («manipulation school»). L'état des recherches actuelles fait apparaître deux facteurs qui, respectivement, justifient et rendent possible une étude de la créativité en traduction. Ce sont, d'une part, l'abandon de la poursuite du fantôme d'une «objectivité »3 mal comprise au profit d'une exigence de «plausibilité interindividuelle» en traductologie, et, d'autre part, l'apport conjoint de différentes sciences parallèles, comme la psychologie et la linguistique cognitive, qui concourent toutes à mieux servir cette «plausibilité interindividuelle» et qui, pour la première fois, font accéder la traductologie au rang de science interdisciplinaire, une interdisciplinarité qui, par beaucoup de chercheurs, est considérée comme un critère sine qua non pour accéder au statut de science.
\end{abstract}

\begin{abstract}
Until very recently it was possible to discard creativity from research in traductology on the basis that it could not be studied scientifically (Gerzymisch-Arbogast 1994: 16). With the destruction of the myth of objectivism (Lakoff with reference to non-occidental countries, Gadamer with reference to the Hermeneutic tradition) and the parallel evolution of traductology towards a taking into consideration of the personality of the translator, "intersubjective plausibility" ("Intersubjektive Nachvollziehbarkeit", Stefanink 1997) has become a basic criterion in the assessment of translation quality. Recent cognitivistic research has fostered this evolution in so far as it has revealed the mechanisms of language management encouraging thus the translator to make use of his associative competence in looking for creative problem-solving within the limits of "intersubjective plausibility" which are fixed by the results of cognitivistic research.
\end{abstract}

MOTS-CLÉS/KEYWORDS

créativité, cognitivisme, compétence associative, plausibilité intersubjective 
[...] theorisation is itself a mode of professional self-defence.

(Pym 1992: 153)

This conception of translation theory is a necessary part of the translator's defensive armor against attacks from the uncomprehending...

(Robinson 1997: 204)

\section{PRÉLIMINAIRES. POURQUOI UNE RÉFLEXION SUR LA CRÉATIVITÉ?}

Idée maîtresse: On reproche au traducteur de «trahir» le texte. Les nouvelles conceptions du texte et de l'acte traduisant autorisent une créativité qui trouve sa légitimation dans la "plausibilité interindividuelle», fondée sur des bases théoriques.

\section{L'accusation de «traîtrise» et la théorie comme auto-défense du traducteur}

\section{«Traduttore - traditore!»}

Lorsqu'on lance cette accusation de traîtrise on ne fait pas dans le détail, mais elle concerne évidemment la créativité du traducteur. On accuse celui-ci de traîtrise dès qu'il s'éloigne tant soit peu des mots du texte source. Si cette accusation a pu se concrétiser en un jeu de mots à circulation internationale, cela est dû à un manque d'arguments théoriques de la part du traducteur pour justifier ses traductions créatives. Les deux citations mises en exergue à cette étude mettent en évidence l'une des fonctions que peut avoir une réflexion théorique sur la traduction face aux accusations de «traîtrise» dont le traducteur a pu être l'objet: l'auto-défense face à l'incompréhension et aux accusations. L'autre fonction importante est celle d'une amélioration de la compétence traduisante suite à une prise de conscience, une prise de conscience qui va de pair avec une "prise d'assurance» (Hönig 1993). En livrant au traducteur les arguments théoriques nécessaires à la plausibilité de sa démarche, cette étude se propose de remplir ces deux fonctions.

\section{Les fondements erronés de l'accusation de traîtrise : la traduction comme copie «fidèle» de l'original}

Si le traducteur a pu être considéré comme un traître, cela tient à deux malentendus: d'une part, une conception erronée de la nature du «texte», d'autre part, une conception erronée de l'opération traduisante. Si Pierre Leyris, citant Yevtouchenko, a pu dire: «Les traductions sont comme les femmes: quand elles sont belles, elles ne sont pas fidèles, quand elles sont fidèles, elles ne sont pas belles », il a manifestement conçu le texte comme une "somme de mots» avec des sens individuels qu'il fallait rendre par les mots correspondants en langue source, et cela à l'image d'une opération traduisante conçue comme une "opération mathématiquement exacte», conformément à l'image de la traduction que se faisait Nida quand il a écrit: «What we do aim at is a faithful reproduction of the bundles of componential features» (1974: 50), le mot étant analysé comme un ensemble de traits sémantiques pertinents comme le préconisait la linguistique structurale. Dans une telle conception de la traduction il n'y avait évidemment pas de place pour la créativité. 


\section{Une nouvelle conception de la traduction}

\subsection{Au niveau de la compréhension du texte: «lectures plurielles» et créativité}

Comme nous le montrerons dans la partie historique, ces conceptions ont subi une évolution fondamentale: le texte est considéré maintenant comme une entité dont le sens dépasse la somme des sens individuels des mots ${ }^{4}$. En outre ce sens ne "réside» pas dans le texte une fois pour toutes. Suite aux réflexions théoriques d'un Roland Barthes ou d'un Umberto Eco, c'est par le récepteur du texte que le sens vient au texte. Ces récepteurs peuvent avoir des «lectures plurielles» du texte. Cela laisse une nouvelle place à la créativité du traducteur déjà au niveau de la compréhension du texte source.

\subsection{Au niveau de la rédaction du texte cible: "skoposthéorie» et différences culturelles comme éléments intégrants de l'acte traduisant}

La skoposthéorie met la finalité de la traduction et les attentes du récepteur du texte cible au premier plan, laissant ainsi une plus grande place à la créativité. Cela a amené les traductologues à se défaire de leur conception de l'acte traduisant comme d'une opération mathématiquement contrôlable et prévisible (un des critères traditionnels dans les sciences dites « exactes» pour accéder au statut de «théorie» dans la conception de Karl Popper ${ }^{5}$, conception dont on est toutefois revenu depuis ${ }^{6}$ ) pour la remplacer par la notion de "plausibilité intersubjective» («Intersubjektive Nachvollziehbarkeit» Stefanink 1997, "Interindividuelle Nachvollziehbarkeit» GerzymischArbogast et Mudersbach 1998): le traducteur doit être en mesure de rendre sa traduction "plausible» à autrui, en expliquant par quels chemins il y est parvenu.

Malheureusement le terme de "Nachvollziehbarkeit» fait partie de ces termes intraduisibles, tels que l'allemand "Gemütlichkeit» ou le roumain «dor», et la traduction par "plausibilité » restera toujours en deçà des implications du terme allemand: alors que «Nachvollziehbarkeit» est un terme motivé et transparent, le terme français reste abstrait, de même le terme allemand reflète une dynamique qui implique une participation active, de la part du récepteur - qui doit revivre au niveau virtuel le parcours effectué par celui dont il doit «nachvollziehen» les actions -, alors que le terme français est statique, le focus du terme français "plausibilité» est sur l'objet, alors que celui de "Nachvollziehbarkeit» est sur le récepteur.

Conclusion: Le but de notre étude est de suivre le cheminement de la pensée créative du traductologue et de rendre ainsi plausibles les solutions créatives qu'il a trouvées à ses problèmes. Cela doit lui permettre de mieux défendre ses choix traduisants. Cette finalité détermine notre conception de la créativité comme une «problem solving activity» (Guilford).

\section{DÉFINITION ET ASPECTS DE LA CRÉATIVITÉ}

Idée maîtresse: L'état actuel de la recherche ne permet pas une définition claire de la créativité, mais elle permet de dégager un certain nombre d'aspects et de critères. 


\section{I.1. La créativité en général}

Dans les différents dictionnaires d'usage général, le terme de créativité garde encore un certain halo de mysticisme. Il est en général défini comme la «capacité de créer» et «créer» est défini comme «action de tirer du néant». Ce vide définitoire transparaît jusque dans les dictionnaires de spécialité les plus récents où «créativité» est défini comme un «concept, encore pas suffisamment circonscrit, pour désigner un ensemble de capacités qui sont à la base de processus créateurs " (Häcker et Stapf 1998: 467; notre trad.). Pour la célèbre traductrice roumaine de Proust et de bien d'autres auteurs français, Irina Mavrodin, la créativité - notamment celle de l'écrivain que fut Proust - est une "épiphanie, suscitée par la mémoire involontaire» (Mavrodin 1994: 126) et relève du hasard.

\section{I.1.1. Les aspects de la créativité}

Les chercheurs en créativité se gardent, eux, de donner une définition et préfèrent parler des «aspects» de la créativité (Brodbeck 1999: 18), comme celui de la «nouveauté » du produit créé et de l'«accueil positif» que lui réserve l'évaluation par les experts (Brodbeck 1999). Après avoir essayé de cerner le problème, le Handbook of Creativity (Glover \& al. 1989: 29) cite le psychologue Claridge (1987: 134) pour exprimer son impuissance à donner une définition claire de la créativité :

I have often felt that as an explanatory concept in psychology, «creativity» has often the qualities of a difficult but persuasive lover, whom reason tells one to abandon yet who continues to satisfy an escapable need.

Et les auteurs du Handbook de conclure:

We cannot define a creative product, but we know it when we see it (Glover 1989: 13).

Une étude des différentes théories en présence permet toutefois de dégager un certain nombre de traits aspectuels sur lesquels les chercheurs en créativité sont d'accord et dont le caractère est pertinent pour le traducteur.

(1) Le produit de l'acte créatif doit être «nouveau»:

No matter what other positive qualities it might possess, we generally insist as a first step that a product be novel before we are willing to call it creative (Jackson and Messick 1967: 4)

(2) Le produit de l'acte créatif doit être «approprié»:

Appropriateness is a crucial conjoint criterion to unusualness. A product must fit the demands of the situation and needs of the creator, and with complex products, the individual parts must form a cohesive whole (Fox 1963: 124).

À côté de ces deux caractéristiques qui font l'unanimité, Jackson et Messick trouvent en outre deux autres critères qui déterminent la qualité des produits de la créativité ainsi que leur degré de créativité : (3) «Transformation of constraint» (que l'on peut appliquer de façon privilégiée au traducteur, qui doit «transformer la contrainte» imposé par le texte source) et (4) «condensation», qui est présente dans des produits qui «do not divulgue their total meaning on first viewing» (1967: 10), c'est-à-dire que le degré de créativité est lié à la condensation du sens et à un certain hermétisme. 


\section{II. ÉTAPES DE LA RECHERCHE EN TRADUCTOLOGIE PAR RÉFÉRENCE À LA CRÉATIVITÉ}

Selon certains chercheurs la "créativité » est un concept à plusieurs degrés (cf. par ex.: Guilford 1950: 446). Examinons les différentes théories de la traduction à la lumière de la place qu'elles réservent aux différents degrés de créativité! Il faut distinguer entre les théories qui se contentent du critère de nouveauté pour parler d'une traduction «créative » et celles qui considèrent la créativité en traduction comme une «problem solving activity» et la soumettent aux deux critères de la «nouveauté» et du caractère «approprié».

\section{II.1. Les théories concevant la créativité comme une "problem solving activity» qui satisfait aux deux critères de la «nouveauté» et du caractère "approprié»}

Idée maîtresse: L'échec de la traduction automatique a initié un débat théorique sur la traduction, dans lequel la place attribuée à la créativité a été de plus en plus importante.

\section{II.1.1. La traduction automatique: le degré zéro de la créativité}

La traductologie en tant que science qui se base sur des recherches menées systématiquement débute avec Weaver, en 1949. Pour Weaver, qui avait travaillé pendant la guerre au décodage des messages secrets ennemis à l'aide d'ordinateurs, traduire c'était décoder; le russe, par exemple était de l'anglais codé:

On se demande naturellement si le problème de la traduction ne pourrait être envisagé comme un problème de cryptographie. Lorsque je regarde un article écrit en russe, je me dis: ceci est réellement rédigé en anglais, mais a été mis en code à l'aide de quelque étrange système de symboles; je vais me mettre à le décoder (cité par DELAVENAY 1959: La machine à traduire, p. 13-14).

Ces espoirs concernant la traduction automatique ont enfermé la réflexion traductologique dans un carcan théorique qui empêchait évidemment toute prise en considération de la créativité. Mounin en est la première victime: son ouvrage sur Les problèmes théoriques de la traduction porte en fait sur les problèmes théoriques posés par la traduction automatique. Le titre de son autre ouvrage traductologique Les belles infidèles - qui porte sur la traduction poétique, dit tout sur la place qu'il attribue à la créativité en traduction: la traduction poétique, qui est évidemment le lieu privilégié de la créativité en traduction, ne peut être qu' «infidèle». Dans cette optique le «traducteur-traître» s'exposait au reproche du crime de lèse-majesté chaque fois qu'il ne reproduisait pas «fidèlement» l'original sacré. La "fidélité» était l'allégeance au texte, un texte compris comme une suite de mots avec chacun un sens bien précis, pour lesquels il fallait trouver les mots correspondants en langue cible, ou comme le formulait $\mathrm{Nida}^{7}$ (1974) du point de vue de la linguistique structuraliste, le mot qui en langue cible reproduisait le plus de traits pertinents du mot de la langue source. 


\section{II.1.2. La stylistique comparée: un premier degré de créativité?}

En dépassant le cadre du mot comme unité de traduction, la stylistique comparée a pu être considérée par certains comme une première étape vers la créativité. Les «transpositions» du type "He swam across the river»= «il traversa la rivière à la nage» constituent effectivement une transformation par rapport aux mots et à la structure de la phrase en langue source. Mais il s'agit là de transformations dues aux contraintes structurales des systèmes linguistiques en regard. La preuve en est que le but de la stylistique comparée a été d'en faire un inventaire complet à des fins de traduction automatique, c'est-à-dire qu'elle les considérait comme des processus automatisables.

\section{II.1.3. Linguistique du texte et créativité}

Dans la mesure où certains courants de la linguistique du texte présentaient le texte comme une "offre d'information», précisant que l'œuvre en elle-même ne "possédait» pas un sens, mais que le sens venait au texte par le récepteur du texte, suite aux théories du sens d'un Roland Barthes ou d'un Umberto Eco, on eût pu attendre une certaine liberté créative pour le traducteur. Mais les linguistes du texte se sont perdus dans une analyse de plus en plus minutieuse du texte qui s'éloignait d'autant plus de la pratique du traducteur qu'elle distinguait une étape bien distincte d'analyse du texte qui était placée avant toute tentative de traduction, interdisant au traducteur de traduire le moindre mot avant d'avoir procédé à une analyse «exhaustive» du texte (pour une critique détaillée de ces théories cf. Stefanink 1996, 1997). L'intuition et la créativité étaient exclues d'emblée de ces recherches étant donné leur «caractère peu accessible à des recherches d'ordre rationnel» (Gerzymisch-Arbogast 1994: 16).

\section{II.1.4. La pragmatique et la créativité}

La place réservée à la créativité par l'approche pragmatique semble plus importante. En effet, la valeur illocutoire des speech acts qui est d'ordre situationnel et ne peut être dégagée à partir d'une analyse sémantique des signifiants individuels qui les composent, oblige le traducteur à trouver les speech acts utilisés dans la langue cible pour exprimer la même valeur illocutoire dans cette même situation. Le "sarut mâna» (litt.: je baise la main) roumain qui est une formule de salutation destinée à la femme (dans certains usages locaux également aux vieilles personnes et aux prêtres), distincte de celle adressée à l'homme, sera traduite par une formule unique en français qui - du moins en synchronie - ne connaît pas cette particularité culturelle. Pourtant il ne s'agit pas là encore, pour nous, d'une manifestation de la créativité du traducteur. Il s'agit de préformés linguistiques situationnels, marqués culturellement, que le bon traducteur bilingue et bi-culturel doit avoir à sa disposition et placer de façon quasi automatique dans le discours de la langue cible. Son choix traduisant ne présentera pas les traits caractéristiques d'une réflexion créative, tels qu'ils ont été dégagés par les chercheurs en créativité, comme par exemple le modèle à quatre phases, qui décrit la progression de la prise de conscience d'un problème jusqu'à sa solution en passant par les phases de "préparation - incubation - illumination évaluation» (cf. Preiser 1976: 42 ss.; Ulmann 1968: 22 ss.). 


\section{II.1.5. Le fonctionnalisme ( Skopostheorie» et la créativité)}

L'approche fonctionnelle, connue sous le nom de «Skopostheorie» (Reiss et Vermeer 1984), situe l'opération traduisante dans le contexte d'une théorie de l'action qui dit que toute action reçoit son sens du but auquel elle tend. Il en découle que c'est la finalité de la traduction qui fournit les critères à respecter. Selon le donneur d'ordres, il peut y avoir changement de la finalité d'un texte ou maintien de cette finalité. Un maintien de la fonction - qui est le cas de loin le plus fréquent - entraîne aussi un maintien de l'effet produit sur le récepteur du texte.

\section{II.1.6. L'herméneutique et la créativité}

Parallèlement au courant « rationnel» qui déclarait arriver au sens à traduire par une analyse minutieuse du texte avant même de permettre la moindre tentative de traduction, s'est développée, sur les traces de Gadamer, une traductologie «herméneutique», qui concevait l'accès au sens par la saisie intuitive de celui-ci, quitte à justifier cette priorité accordée à la créativité dans l'accès au sens, par une analyse postérieure. Paepcke et Forget (1981) parlent de l'intuition foudroyante, par laquelle arrive l'heureuse trouvaille.

Conclusion: C'est avec la Skopostheorie qu'intervient le type de créativité qui nous intéresse! Face à l'exigence du maintien de l'effet produit sur le récepteur, le traducteur doit prendre en considération les éventuels écarts entre les deux cultures en présence et développer une créativité proportionnelle à cet écart. L'approche herméneutique, quant à elle, accorde à la créativité la place qui lui revient du point de vue épistémologique. Reste à savoir ce qui se cache derrière des notions comme «intuition foudroyante» que nous essayerons de démystifier en cherchant des modèles explicatifs dans les sciences voisines.

\section{II.2. Les théories de la créativité en traduction qui ne prennent en considération que le critère de «nouveauté»}

Idée maîtresse: Les théories qui ne prennent pas en considération les deux critères d'évaluation que sont la «nouveauté» du produit et son caractère «approprié » et qui ne considèrent pas la créativité comme une "problem solving activity» ne sont pas conciliables avec la déontologie du traducteur.

\section{II.2.1. Le texte source comme source d'inspiration créatrice}

Il existe des conceptions de la traduction qui considèrent l'œuvre à traduire comme une stimulation de l'impulsion créatrice chez le traducteur. "Les belles infidèles ", comme on appelle les productions de ce genre au XVIII ${ }^{\mathrm{e}}$ siècle, qui ne se servent du texte source que comme d'un tremplin pour laisser libre cours à l'imagination du «traducteur» (qui de ce fait devient «auteur»), en sont un exemple historique. À notre époque, on retrouve cette conception dans les pays anglo-saxons: d'une part aux États-Unis, dans les «Translation and Writing Workshops», notamment le célèbre «workshop» de l'université d'Iowa, où, les mots d'un poème à "traduire» ne sont considérés que comme des indicateurs de sens, que le traducteur doit saisir intuitive- 
ment et dont il doit s'inspirer pour créer son œuvre à lui en langue cible; d'autre part chez les tenants de la «manipulation school» - dont l'un des fondateurs, James Holmes, est issu de ces «translation workshops»-qui, partant de la constatation que toute traduction est déjà "manipulation» du texte source, ne se soucie plus de la conformité du texte cible avec le texte source, mais considère qu'il suffit qu'un traducteur déclare son texte comme une «traduction» pour que l'on ne discute plus s'il a rajouté ou changé des choses selon l'inspiration déclenchée par sa saisie du texte source. Ils publient leurs idées dans un volume intitulé: The Manipulation of Literature: Studies in Literary Translation (Hermans 1985).

\section{II.2.2. Les dévoiements de la licence créatrice en traduction}

Cette licence créatrice a pu aboutir à des utilisations de la traduction à des fins politiques ou idéologiques, comme celle de la "post-colonial translation» qui déclare vouloir rétablir les injustices créées par les mesures imposées aux traducteurs sous l'occupation coloniale, ou encore la «traduction féministe», qui, elle, veut rétablir les injustices commises envers les femmes et « déclare la guerre au vieux concept binaire de la bi-polarisation entre le texte source et la traduction» (Bassnet 1991: 66) "pour la remplacer par une "créativité" au service d'une idéologie: "Translation is not a carrying across, but a reworking of meaning» (Simon 1996: 23, cite Godard) et "Translation must be seen as a fluid production of meaning, similar to other kinds of writing» (12; nous soulignons). Cette "créativité» va jusqu’à vouloir «re-sexuer» (20) le langage (ex: orthographe «auther» pour «author» lorsqu'il s'agit d'une femme écrivain, traduction de «aube» par «dawn» mais reprise de «dawn» par le pronom personnel «she» etc.). Tout cela avec une intention déclarée: «My translation is a political activity, aimed at making language speak for women. So my signature on a translation means: this translation has used every translation strategy to make the feminine visible in language» (15; Simon cite Lotbinière Harwood).

\section{II.2.3. Une créativité au service de la traduction}

Ces conceptions de la créativité ne sont guère compatibles avec la déontologie du traducteur, qui est redevable au texte source pour le message à communiquer. Il ne peut s'agir pour lui de «tirer du néant» (cf. définition du Petit Robert à l'article «créer»). Les recherches empiriques que nous avons menées au moyen d'analyses conversationnelles ethnométhodologiques s'inscrivent parfaitement dans la conception de la créativité - comprise comme une activité cherchant des solutions à des problèmes - telle qu'elle est défendue par le psychologue Guilford et son école (Guilford 1971:312-345 consacre un chapitre entier aux liens entre "Problem solving and creative production »): «There is something creative about all genuine problem solving, and creative production is typically carried out as a means to the end of solving some problem» (Guilford 1971:312).

Il s'agit d'une créativité mise au service de la traduction et non pas d'une traduction, dite «créative», au service d'une idéologie.

Conclusion: La créativité qui nous intéresse se situe entre, d'une part, les dévoiements incontrôlés de théoriciens comme ceux de la «manipulation school», et d'autre 
part les «transpositions» de la stylistique comparée que certains considèrent déjà comme un premier pas dans la créativité, mais qui nous semblent encore beaucoup trop déterminées par les structures des langues en présence.

\section{LES DIFFÉRENTES TENDANCES ACTUELLES DE LA RECHERCHE EN CRÉATIVITÉ, DE LA RECHERCHE SUR LA MÉMOIRE AINSI QUE DE LA LINGUISTIQUE COGNITIVE ET LEUR PERTINENCE POUR L'ÉTUDE DE LA CRÉATIVITÉ EN TRADUCTOLOGIE}

Idée maîtresse: Les recherches dans ces trois domaines respectifs se soutiennent et se complètent mutuellement pour fournir des modèles descriptifs autant qu'explicatifs au phénomène de créativité.

\section{III.1. Les modèles descriptifs de la créativité}

Bien que chaque description ait déjà un caractère explicatif, on peut distinguer entre des modèles qui sont plutôt descriptifs face à d'autres qui sont en outre explicatifs.

\section{III.1.1. Les enchaînements associatifs ( chaining») chez le linguiste (cognitif) Lakoff}

Pour mesurer le degré de créativité d'un individu Mednick (1962) avait développé le «Remote Associates Test»(RAT) (Test des associations à distance) qui consiste à trouver des associations communes face à des mots qui, à première vue, n'ont rien en commun et sont sémantiquement très éloignés les uns des autres. Ainsi «out, dog, cat» ont en commun "house»; "rat, blue, cottage» ont en commun "cheese». Schank (1982) expliquera comment la façon dont notre mémoire stocke notre vécu est responsable de ces «remote associations».

Ces tests ne sont pas sans rappeler les enchaînements associatifs que Lakoff (1987) découvre dans la métaphorique populaire («folk theory», «folk models of categorization» Lakoff 1987: 121). Lakoff a réussi à mettre en évidence les enchaînements associatifs («chaining») qui conditionnent notre vie quotidienne: «[...] metaphors that structure the ordinary conceptual system of our culture, which ist reflected in our everyday language» (Lakoff et Johnson 1980: 139).

Il nous montre, par exemple, comment nous associons les concepts de «anger» et «lust» par le biais de représentations métaphoriques qui ont certains points communs. Dans l'imagination populaire, en effet, «anger» est associé à «feu» comme en témoignent les citations suivantes: Those are inflammatory remarks. He was breathing fire. Your insincere apology just added fuel to the fire. After the argument, Dave was smoldering for days, etc. (Lakoff 1987: 388). La même idée de feu se retrouve dans le scénario de «lust»: She is an old flame. He was consumed by desire. She's hot stuff. Hey, baby light my fire, etc. (Lakoff 1987: 410). Lakoff en conclut que, par le biais de ces représentations métaphoriques, les deux catégories «anger» et «lust» peuvent se trouver associées. 


\section{III.1.2. La pensée «latérale» / «divergente»}

Cette approche associationniste est exploitée par les chercheurs en créativité qui considèrent la créativité comme une «problem solving activity». Ainsi ce que Guilford appelle la pensée «divergente» est une stratégie pour résoudre des problèmes pour lesquels la pensée «convergente», logique, n’a pas trouvé de solutions. Elle consiste à examiner les problèmes sous un autre aspect, à les aborder d'un autre point de vue. De Bono a préféré le terme de «pensée latérale» par opposition à la pensée logique qu'il appelle «verticale». Il s'agit là d'une stratégie créative fondamentale qui permettra au traducteur de trouver des solutions en reportant son attention sur d'autres éléments scéniques marginaux dans la culture source mais prototypiques dans la culture cible (stratégie soutenue par le recherches de Fillmore, de Langacker et de la sémantique des prototypes, exposées plus bas).

\section{III.1.3. Eleanor Rosch et la sémantique des "prototypes»}

La psychologue Eleanor Rosch (1973) nous amène à faire le lien entre la recherche en créativité et la linguistique cognitive. Elle montre que la vue structuraliste des catégories sémantiques bien déterminées, avec des traits pertinents nettement définis entre les différents éléments d'une catégorie, est une projection des scientifiques. En effet, notre découpage du monde en catégories sémantiques est fonction de notre vécu, de nos expériences ${ }^{8}$. La catégorie oiseau, par exemple, comprend des éléments plus représentatifs que d'autres de cette catégorie. Et cette représentativité varie avec l'expérience vécue au quotidien. Pour un Européen, le moineau pourrait être un représentant prototypique de la catégorie oiseau, alors que pour un habitant du désert africain cela pourrait être l'autruche, le pingouin étant pour les deux un élément marginal de cette catégorie, appartenant par certains de ses traits à la catégorie animal aquatique.

Le traducteur du Notre Père qui aura traduit le «pain quotidien» par le «bol de riz» n'aura donc fait que traduire l'élément prototypique dans la civilisation source par l'élément prototypique correspondant dans la civilisation cible.

\section{III.1.4. Le "figure/ground alignment» de Langacker}

En tant qu'individu bilingue et bi-culturel le traducteur aura ainsi changé de focus, il aura centré son attention sur un autre élément de ce champ sémantique de nourriture quotidienne. Car comme nous l'apprend la linguistique cognitive de Langacker (1987: 120) notre perception est toujours organisée selon un «figure/ground alignment», c'est-à-dire que nous percevons toujours un élément («figure») qui se détache du reste (le "ground alignment»). Selon Langacker cette

figure/ground organisation is not in general automatically determined for a given scene; it is normally possible to structure the scene with alternate choices of figure. However, various factors contribute to the naturalness and likelihood of a particular choice (Langacker 1987: 120).

Si l'on comprend les «différents facteurs» qui influencent «un choix particulier» comme étant les facteurs culturels, on saisit le potentiel explicatif de cette théorie, qui 
vient renforcer ce qui a été dit en III.1.3 à propos des prototypes culturellement marqués. L'importance de cette observation pour le traducteur traduisant dans le cadre de la Skopostheorie est capitale, comme nous le verrons.

La notion de «scene» utilisée par Langacker nous oblige à situer la théorie du «figure/ground alignment» dans le cadre plus vaste de la «scenes-and-framessemantic» de Fillmore.

\section{III.1.5. La sémantique des "scenes-and-frames» de Charles Fillmore}

Partant de la constatation que l'on ne comprend que par rapport à ce que l'on sait déjà, c'est-à-dire par rapport à son world knowledge, Fillmore présente l'acte de compréhension d'un texte comme étant lié, d'une part, aux mots de ce texte (y compris les règles grammaticales qui les relient) - qui constituent les linguistic frames - et, d'autre part, au vécu du récepteur du texte, c'est-à-dire aux différentes expériences vécues qu'il a stockées dans sa mémoire longue et qu'il appelle dans sa mémoire active au contact de ces mots, se fabriquant ainsi des cognitive scenes. Le "frame» dans le texte provoque une «scene» chez le récepteur.

Une «scene» est composée d'éléments scéniques qui peuvent être - ceci rejoint la notion d'éléments prototypiques de Rosch - centraux ou marginaux. Les éléments d'une même "scene» sont reliés associativement entre eux par le fait d'appartenir à cette «scene». Comme nous allons le montrer dans l'exemple donné plus bas, le traducteur qui se trouvera devant le problème d'un vide lexical en langue cible sera créatif si par association, il traduit par un autre élément de cette «scene» qui sera prototypique en culture cible et, de ce fait, lexicalisé (cf. l'exemple de "potty chair» plus bas).

\section{III.2. Un modèle explicatif: La «mémoire dynamique» de Roger Schank - "Memory Organisation Packets» (MOPs) et «Thematic Organisation Points» (TOPs)}

Les recherches de Roger Schank sur la mémoire nous permettent de trouver un modèle explicatif aux modèles descriptifs que nous venons de présenter.

Schank étudie la façon dont nos souvenirs sont reliés les uns aux autres. Il nous décrit la scène suivante:

Hier j'étais chez le médecin. J'étais en train de lire un journal dans la salle d'attente et je constatai qu'un patient qui était venu après moi, a été traité avant moi. Sans doute la facture du médecin sera de nouveau trop élevée (Schank 1982: 85; notre traduction).

Schank se demande quels sont les rapports entre la facture trop élevée et le fait qu'un autre patient passe avant lui. L'élément commun est manifestement l'expérience d'injustice que le patient a faite à une occasion antérieure et qu'il refait à cette occasion. Cette expérience a fait partie de la scène "facture de médecin », qui fait ellemême partie du scénario plus vaste de «visite médicale». Si elle a pu ressurgir à cette occasion, dans un autre contexte d'une "visite médicale», c'est qu'elle a dû être stockée comme un élément détaché de la scène «facture de médecin», dans laquelle elle a été vécue la première fois ${ }^{9}$ : l'élément scénique commun qui rapproche les deux «scenes» est dans ce cas le vécu de l'injustice. 
Schank conclut que nos expériences - le vécu sur le fond duquel s'effectue tout processus de compréhension - sont stockées par petits paquets, décomposés en éléments et isolés de leur contexte scénique, qu’il appelle «Memory Organisation Packets» (MOPs), et que nos associations ne sont pas l'effet du hasard, mais suivent des voies déjà tracées dans notre mémoire. Ce stockage se fait indépendamment de la scène vécue, ce qui explique la résurgence de ces "paquets» dans une autre scène qui présente certains éléments communs aux deux scènes.

Mais il existe des associations qui ne s'expliquent pas par cette appartenance commune d'un MOP à différentes «scenes». Schank les explique par des structures de notre mémoire plus abstraites, à un niveau supérieur: les «Thematic Organisation Points"(TOPs). Le lien qui permet d'associer Romeo and Juliet et West Side Story est d'ordre structural: l'action a la même structure, la même «Gestalt». Dans les deux cas il s'agit de deux amants qui veulent s'unir et qui doivent lutter contre la résistance que leur oppose le monde environnant (les parents dans le premier cas, les «gangs» dans le deuxième). La structure commune est constituée par le même but et le même type de conditions (Schank 1982: 113). C'est ce type de structure commune qui permettra de traduire par exemple un proverbe par un autre proverbe, comme porter de l'eau à la rivière qui pourra être rendu par l'allemand Eulen nach Athen tragen ou l'anglais to carry coal to Newcastle. Dans l'exemple suivant, où il fallait traduire un passage sur les bienfaits du vin, le texte comportait une citation de Keats sur le vin: "Oh, for a beaker of the warm south,/ Full of the true, the blushful Hippocrene,/ With beaded bubbles winking at the brim” sighed Keats. » Les étudiants qui devaient le traduire ont convenu après quelque discussion qu'il ne fallait pas traduire Keats, mais choisir une autre citation. En effet, une des fonctions de la citation de Keats, dans le texte anglais, est de créer une connivence entre l'auteur et le lecteur, en montrant que tous deux participent de la même culture littéraire. L'autre fonction était de vanter les mérites du vin. Le texte était tiré d’un journal anglais et la traduction destinée à un public de lecteurs de journal équivalent en Allemagne, qui n'avait certainement pas la même intimité culturelle avec Keats. On a convenu de le remplacer par une citation de la Bible, le psaume 104, 15: "Le vin réjouit le cour de l'homme", comme il est déjà écrit dans la Bible.» Les deux citations ont la même finalité de faire l'éloge du vin, la citation de la Bible sera plus connue du public cible et recréera plus aisément cette connivence ${ }^{10}$.

\section{LES DIFFÉRENTS MODÈLES THÉORIQUES ET LEUR POTENTIEL DESCRIPTIF ET EXPLICATIF À L'ÉPREUVE DE LA PRATIQUE}

Pour examiner cette question, nous allons prendre un exemple de traduction «créative $^{11} »$. Il s'agit d'un texte en anglais sur les problèmes des couples où la femme et l'homme travaillent tous deux, ce qui leur pose des problèmes pour gérer à la fois leur vie professionnelle et l'éducation des enfants. La phrase à traduire était: "They had difficulties to juggle two careers and a potty chair» (littéralement: Ils avaient des difficultés à gérer deux carrières et la chaise percée pour enfants).

On a demandé à des étudiants allemands en traductologie, section d'anglais, de traduire ce texte en allemand. Pour «to juggle», qui exprime la faculté de gérer deux choses en même temps, la locution métaphorique « unter einen Hut bringen » (littéralement: mettre sous un même chapeau) s'impose. Le lexème potty chair n'existant 
pas en allemand certains ont traduit relativement littéralement en prenant le correspondant le plus proche Kindertöpfchen (pot de chambre pour enfants) l'utilisant en collocation avec unter einen Hut bringen. Cette collocation est impossible à cause de la valeur littérale qu'elle donne à la locution métaphorique unter einen Hut bringen: on voit littéralement le pot de chambre sous le chapeau. D'autres ont trouvé une solution créative en traduisant:

(1) ... Kind und Karriere unter einen Hut zu bringen.

(...gérer simultanément enfant et carrière.)

(2) ... zwei Karrieren und Windelwechseln unter einen Hut zu bringen

(...gérer simultanément deux carrières et l'action de changer les couches.)

Ni Kind ni Windelwechseln ne sont évidemment la traduction de potty chair. Mais ces traductions ne choquent pas le lecteur allemand. Elles sont «appropriées». Elles font partie de la «scene» «soins de l'enfant», telle qu'elle est ancrée dans le vécu, et donc dans la mémoire longue de l'Allemand prototypique. Quelque chose a dû permettre le rapprochement entre ces mots, nihil ex nihilo. Examinons ces traductions à la lumière des modèles explicatifs que nous fournissent les différentes approches théoriques!

La sémantique des prototypes vient compléter la description du processus de compréhension fournie par Fillmore dans sa «scenes-and-frames-semantics», permettant ainsi une première approche explicative à la créativité de ces traductions. Comme nous l'avons vu plus haut, les éléments des différentes catégories sémantiques ne sont pas représentatifs (prototypiques) au même degré de la catégorie sémantique à laquelle ils appartiennent. Leur caractère prototypique peut varier selon les cultures: si, pour un Européen, le moineau peut être prototypique de la catégorie «oiseau», il ne le sera plus pour un Africain qui pensera peut-être plutôt à l'autruche, considérant que le trait distinctif (prototypique) de l'oiseau ne réside pas forcément dans sa «capacité de voler» mais dans le fait de posséder des «ailes». Le caractère prototypique du vécu stocké dans la mémoire est fonction de la fréquence au quotidien de ce vécu. Malgré sa «capacité de voler» et le trait pertinent des "ailes», le vécu collectif associera la poule plutôt avec un bipède en train de picorer dans la basse-cour (à qui ses «ailes» ne permettent même pas de s'envoler pour échapper au paysan qui la poursuit pour la mettre "au pot») ou avec l'image (culturellement marquée) de «la poule au pot», gravée dans la mémoire des Français en association avec Henri IV, et faisant inévitablement partie de leur vécu scolaire ${ }^{12}$.

Dans notre exemple le mot («frame») potty chair a déclenché la visualisation d'une «scene » à laquelle on pourrait donner le nom de «soins du bébé» ou soins de l'enfant en bas âge, ou encore, à un niveau supérieur, "éducation de l'enfant». En traduisant potty chair par «l'action de changer les couches», dans l'exemple (2), les traducteurs ont choisi un autre élément de la «scene» qu'on peut intituler «éducation de l'enfant». Il faut penser que, en raison de leur bi-culturalisme, ils ont - consciemment ou inconsciemment - choisi un élément qui était prototypique de cette «scene» dans la culture de la langue cible. Le fait que potty chair soit lexicalisé en anglais, mais non pas en allemand semble appuyer l'hypothèse qu'il s'agit d'un élément prototypique en anglais au contraire de l'allemand, où c'est l'action de «changer les couches» qui semble être une activité prototypique de cette «scene».

La sémantique des prototypes n'explique cependant pas la traduction (1). En traduisant par Kind, qu'il faut comprendre ici comme une ellipse sémantique de 
Kindererziehung (éducation de l'enfant), les traducteurs ont choisi de traduire par un scénario plus vaste et plus abstrait qui englobe plusieurs «scenes» de l'éducation de l'enfant. Là ce sont les TOPs de Schank qui permettent d'expliquer l'association à un niveau supérieur.

\section{CONCLUSION}

Cette présentation a mis en évidence les résultats d'un faisceau de recherches qui, toutes, concourent à décrire et à expliquer les solutions créatives que peut trouver un traducteur aux problèmes qui lui sont posés, les légitimant en les rendant plausibles ( nachvollziehbar»). Les étapes de la créativité peuvent se résumer comme suit.

Le texte source se présente comme un «frame» (linguistique) qui déclenche une «scene» (cognitive) dans l'esprit du traducteur (Fillmore 1976). Dans cette «scene» il y a des éléments centraux, " prototypiques» (Rosch), suivant le principe du «figure/ ground alignment» (Langacker 1987). Le traducteur rencontre un problème de traduction, si cette relation d'ordre prototypique entre le «figure» et le "ground alignment» n'est pas la même dans la culture cible. Il doit alors focaliser différemment les éléments de la "scene» et choisir un autre élément ("potty chair» devient «Windelwechseln», c'est-à-dire «changer les couches»). Changeant ainsi l'angle sous lequel il envisage le problème, le traducteur fait appel à ce que De Bono (1970) et Guilford (1950) ont appelé respectivement la «pensée latérale» et la "pensée divergente». La solution créative qu'il trouvera devra être non seulement «nouvelle», mais encore «appropriée» (Fox 1963: 124) pour satisfaire au critère du «maintien de l'effet produit» (Reiss et Vermeer (1984) : «Wirkungsgleichheit») exigée par la déontologie du traducteur. Une démarche ainsi conçue permet d'obtenir des traductions « intersubjectivement» (Stefanink 1997) ou «interindividuellement» (GerzymischArbogast et Mudersbach 1998) «plausibles», en conformité avec les critères d'évaluation préconisés par une traductologie basée sur la pratique.

On le voit, la créativité connaît des fondements théoriques qui permettent de suivre son cheminement et de le justifier, celui-ci devenant ainsi «nachvollziehbar». En traduction ceci vaut tout particulièrement pour une créativité conçue comme une "problem solving activity» (Guilford 1950) et un nouveau critère d'évaluation qui est la «intersubjektive Nachvollziehbarkeit», la "plausibilité interindividuelle» (Stefanink 1997). Cela ne réduit-il pas singulièrement le rôle du «hasard» qui, d'après certains chercheurs, est le déclencheur - incontrôlable et inexplicable - de la créativitét ${ }^{13}$ ?

Les hypothèses que nous venons de formuler sur les processus mentaux qui mènent à la solution créative d'un problème se situent dans la ligne de pensée d'un Karl Popper (1935), qui dit à propos du hasard que celui-ci connaît, lui aussi, des lois, que notre niveau de connaissances ne permet malheureusement pas de connaitre suffisamment au point d'en déduire des prédictions - qui pour Popper sont la condition nécessaire au statut de science -, mais que la tâche du chercheur est de faire reculer les limites du hasard - qui n'est pour Popper qu'un terme pour désigner ce qui est imprévisible et par conséquent non scientifique - en essayant de découvrir ses lois et de les décrire. Dans la vision de Popper, les recherches que nous venons de présenter permettent d'asseoir plus solidement le statut scientifique de la traductologie, dont on connaît la précarité. 
En ce qui concerne le théoricien, les recherches que nous venons de présenter lui permettront d'asseoir plus solidement le statut scientifique de sa discipline, en satisfaisant un peu plus aux critères énoncés par Popper. Elles lui fourniront également des arguments dans la discussion sur la notion de "fidélité» si centrale en traductologie. En ce qui concerne le praticien, la compréhension de ces phénomènes doit déculpabiliser le traducteur face aux reproches de «traîtrise» («Traduction - Trahison!») et l'encourager à faire confiance à ses intuitions, évaluées au regard de ces théories. Quant au didacticien, ces réflexions lui fourniront les bases théoriques nécessaires pour donner à ses étudiants le «courage»- une des conditions pour la créativité selon les chercheurs en créativité - d'aller jusqu'au bout de leurs intuitions créatives.

\section{NOTES}

1. Je remercie la fondation «Toepfer» (Hambourg) pour la bourse, grâce à laquelle il m’a été possible de mener à bien ces recherches.

2. Cf. par ex. : Berman (1985: 140) : «Pour traduire, le traducteur doit chercher inlassablement le nonnormé de sa langue. (...) La traduction c'est cela: chercher-et-trouver le non-normé de la langue maternelle pour y introduire la langue maternelle et son dire.» Nous ne partageons toutefois pas cette position outrée de l'aspect créatif dans l'activité traduisante, puisqu'elle ne prend en considération qu'un des aspects de la créativité telle qu'elle est décrite par les chercheurs en créativité (cf. plus bas I.1.1.)

3. Voir à ce propos, par exemple, le chap. 26 de Lakoff /Johnson (1982: 195) intitulé «The Myth of Objectivism in Western Philosophy and Linguistics» : «Meaning, therefore, is never disembodied or objective and is always grounded in the acquisition and use of a conceptual system» (1982: 197).

4. Stolze (1992) parle de la «Übersummativität» du texte, qui signifie le dépassement de la somme des mots.

5. Le «falsificationism» de Popper (1935) veut qu'une théorie qui n'est pas en mesure de faire des prédictions explicites ne peut pas être falsifiée et n'est par conséquent pas scientifique.

6. Cf. Conant 1947, Lakatos 1970

7. «What we do aim at is a faithful reproduction of the bundles of componential features» (Nida 1974: 50).

8. Lakoff et Johnson $(1980: 211)$ parlent des « conventional metaphorical concepts we take as structuring our everyday conceptual system ».

9. On retrouve ici chez Schank les idées énoncées par Spearman (1931) cinquante ans plus tôt.

10. L’exemple est tiré de Kußmaul (2000).

11. Exemple de Kußmaul (2000).

12. Le fait qu'il s'agit là de "folk models of categorization ", comme dit Lakoff, qui sont basés sur le vécu et sur la perception de la réalité qui en découle, et peuvent par conséquent varier d'une culture à l'autre, est souligné par le fait que, par exemple, les Français et les Allemands distinguent une souscatégorie «volaille» et "Geflügel» alors que pour les Roumains tout rentre dans une même catégorie «oiseau» avec le mot «pasare».

13. Ainsi Paepcke et Forget (1981) parlent de «l'intuition foudroyante», Mavrodin (1994) écrit un livre sur La main qui écrit, ayant pour sous-titre: Une poïétique du hasard, et présentant la créativité comme quelque chose d'inexplicable.

\section{RÉFÉRENCES}

BAssnet, S. (1991): Translation Studies, Revised Edition, London, Routledge.

Berman, A. (1985): «La traduction et la lettre - ou l'auberge du lointain», Les tours de Babel, T.E.R.

Brodbeck, K.-H. (1999) : Entscheidung zur Kreativität, Darmstadt, Primus Verl.

Claridge, G. (1987) : "Psychotism and Arousal", in Personality dimensions and arousal, J. Strelau and H. J. Eysenck (Eds.), New York: Plenum Press. 
Conant, J.B. (1947): On Understanding Science, New Haven, Yale University Press.

De Bono, E. (1970): Lateral Thinking. A Textbook of Creativity. London, Ward Lock Educational. Delavenay, G. (1959): La machine à traduire, Paris, P.U.F.

Fillmore, Ch. J. (1976): "Scenes-and-Frames Semantics", in Linguistic Structures Processing, Antonio Zampolli (dir.), Amsterdam, N. Holland, p. 55-88.

Fox, H.H. (1963): "A Critique on Creativity in Science", in Essays on Creativity in the Sciences, M.A. Coler (Ed.), New York, New York University Press, p. 123-152.

Gerzymisch-Arbogast, H. (1994): Übersetzungswissenschaftliches Propädeutikum. Grundlagen $z$ u einer wissenschaftlichen Methodik des Übersetzens, Tübingen, Francke (UTB 1782).

Gerzymisch-Arbogast, H. et K. Mudersbach (1998): Methoden des wissenschaftlichen Übersetzens, Tübingen, Francke (UTB 1990).

Glover, A. J. et al. (dir.) (1989): Handbook of Creativity, New York, Plenum Press.

Guilford, J. P. (1950): “Creativity”, American Psychologist, 5, p. 444-454.

Guilford, J. P. (1971): The Nature of Human Intelligence, London, McGraw-Hill.

Guilford, J. P. (1977): Way beyond the QI, Buffalo, Creative Education Foundation.

Häcker, H. et K. H. Stapf (dir.) (1998): Dorsch Psychologisches Wörterbuch, Göttingen: Hans Huber Verlag.

Hermans, T. (ed.) (1985): The Manipulation of Literature. Studies in Literary Translation. London/Sydney, Croom Helm, New York, St. Martin's Press.

HöNIG, H. G. (1993) : «Vom Selbst-Bewußtsein des Übersetzers», dans Traducere Navem. Festschrift für Katharina Reiß zum 70. Geburtstag, J. Holz-Mänttäri et C. Nord (eds.) (Studia translatologica ser. A vol. 3), Tampere, Schriften des Instituts für Translationswissenschaft der Universität Tampere, p. 77-90.

Kussmaul, P. (2000): Kreatives Übersetzen, Tübingen, Stauffenburg.

Jackson, P.W. et S. Messick (1967): "The Person, the Product, and the Response: Conceptual Problems in the Assessment of Creativity", dans Creativity and Learning, J. KAGAN (Ed.), Boston, Houghton Mifflin, p. 1-19.

Lakatos, I. (1970): "Falsification and the Methodology of Research Programmes", dans Criticism and the Growth of Knowledge, I. Lakatos and A. Musgrave (Eds.), Cambridge: Cambridge University Press: p. 8-101.

Lakoff, G. (1987): Women, Fire and Dangerous Things, What Categories Reveal about the Mind, Chicago, University of Chicago Press.

Lakoff, G. et M. Johnson (1980): Metaphors we live by, Chicago, The University of Chicago Press.

LANGACKeR, R. W. (1987): Foundations of Cognitive Grammar, Stanford, Stanford University Press. Mavrodin, I. (1994): Mâna care scrie. Spre o poetica a hazardului, Bucarest, Editura Eminescu.

Mednick, S. A. (1962): “The Associative Basis of the Creative Process", Psychological Review, 69, p. 220-232.

NidA, E. (1974): "Semantic Structure and Translating", p. 50, dans Aspekte der theoretischen sprachbezogenen und angewandten Übersetzungswissenschaft II, W.Wilss et G. Thome, Heidelberg, Groos, 1974.

Paepcke, F. et P. Forget (1981): Textverstehen und Übersetzen. Ouvertures sur la Traduction, Heidelberg, Groos.

Popper, K. R. (1935; trad. 1959): The Logic of Scientific Discovery, New York, Basic Books.

Preiser, S. (1976) : Kreativitätsforschung, Darmstadt, Wissenschaftliche Buchgesellschaft.

Pyм, A. (1992): Translation and Text Transfer: An Essay on the Principles of Intercultural Communication, Frankfurt/Main: Peter Lang.

Reiss, K and H. Vermeer (1984): Grundlegung einer Translationstheorie, Tübingen, Niemeyer.

Rickheit, G. and H. Strohner (1993): Grundlagen der kognitiven Sprachverarbeitung. Modelle, Methoden, Ergebnisse, Tübingen, Francke.

Robinson, D. (1997) : Becoming a Translator, London, Routledge.

Rosch, E. (1973): “Cognitive Psychology”, Cognitive Psychology, 4, p. 328-350. 
Schank, R. C. (1982): Dynamic Memory. A Theory of Reminding and Learning in Computers and People, London, New York, Cambridge University Press.

Spearman, C. (1931): Creative Mind, New York, Appleton.

Simon, S. (1996): Gender in Translation: Cultural Identity and the Politics of Transmission, London and New York, Routledge.

Stefanink, B. (1996): Compte rendu de Heidrun Gerzymisch-Arbogast: Übersetzungswissenschaftliches Propädeutikum. Grundlagen zu einer wissenschaftlichen Methodik des Übersetzens, Tübingen, Francke, 1994, dans Info-DaF, 2/3, p. 278-281.

Stefanink, B. (1997) : "“Esprit de finesse" - "Esprit de géométrie": Das Verhältnis von "Intuition" und "übersetzerrelevanter Textanalyse" beim Übersetzen», dans Linguistik und Literaturübersetzen, Rudi Keller (éd.), Tübingen, Narr, p. 161-184.

Stolze, R. (1992): Hermeneutisches Ubersetzen, Tübingen: Narr.

Ulmann, G. (1986): Kreativität. Neue amerikanische Ansätze zur Erweiterung des Intelligenzkonzepts, Weinheim, Beltz.

Weaver, W. (1949/1955): "Translation", dans Machine Translation of Languages, W. N. Locke et A. D. Воотн (dir.), Cambridge, MIT Press. 